\title{
Surfaces
}

\section{Sifflements dans l’obscurité. L’événementialité de Bill Readings}

\section{Johanne Villeneuve}

Volume 6, 1996

LES ÉCONOMIES DISCURSIVES DU SAVOIR ET DE LA CULTURE DANS LE SILLAGE DE L'OEUVRE DE BILL READINGS

THE DISCURSIVE ECONOMIES OF KNOWLEDGE AND CULTURE, WITH CONSTANT REFERENCE TO THE WORK OF BILL READINGS

URI : https://id.erudit.org/iderudit/1064854ar

DOI : https://doi.org/10.7202/1064854ar

Aller au sommaire du numéro

Éditeur(s)

Les Presses de l’Université de Montréal

ISSN

1188-2492 (imprimé)

1200-5320 (numérique)

Découvrir la revue

Citer cet article

Villeneuve, J. (1996). Sifflements dans l'obscurité. L'événementialité de Bill Readings. Surfaces, 6. https://doi.org/10.7202/1064854ar
Résumé de l'article

Partant du travail de Bill Readings et de sa critique de l'université, cet article s'interroge sur la possibilité pour les intellectuels actuels d'être mobiles et ludiques dans la circulation des savoirs. Dans un premier temps, la critique et les solutions stratégiques offertes par Bill Readings rendent nécessaires un retour à la théorie de l'événement (Lyotard, Veyne, Koselleck, etc.); dans un deuxième temps, elles nous forcent a nous demander si, malgré le principe de « jeu » dont elles se réclament, ces stratégies échappent totalement a l'idéologie, le jeu pouvant aussi contenir la possibilité de sa propre tyrannie.
Copyright (c) Johanne Villeneuve, 1996

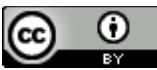

Ce document est protégé par la loi sur le droit d'auteur. L'utilisation des services d'Érudit (y compris la reproduction) est assujettie à sa politique d'utilisation que vous pouvez consulter en ligne.

https://apropos.erudit.org/fr/usagers/politique-dutilisation/ 


\section{Sifflements dans \\ l'obscurité \\ L'événementialité de Bill Readings}

Johanne Villeneuve

Département de littérature comparée

Université de Montréal

villenj@ere.umontreal.ca

Surfaces Vol. VI. 209 (v.1.0F - 20/12/1996) - ISSN:

1188-2492

Tout texte reste la propriété de son auteur. Néanmoins, Surfaces demande d'être citée à l'occasion de toute autre publication du texte en question.

\section{RÉSUMÉ}

Partant du travail de Bill Readings et de sa critique de l'université, cet article s'interroge sur la possibilité pour les intellectuels actuels d'être mobiles et ludiques dans la circulation des savoirs. Dans un premier temps, la critique et les solutions stratégiques offertes par Bill Readings rendent nécessaires un retour à la théorie de l'événement (Lyotard, Veyne, Koselleck, etc.); dans un deuxième temps, elles nous forcent a nous demander si, malgré le principe de jeu dont elles se réclament, ces stratégies échappent totalement a l'idéologie, le jeu pouvant aussi contenir la possibilité de sa propre tyrannie.

\section{ABSTRACT}

Taking its point of departure from Bill Readings's work and from his critique of the university, this article examines the possibility of contemporary intellectuals' mobility and 
playfulness in circulating knowledge. First, the criticism and strategic solutions offered by Bill Readings prompt a return to the theory of the event (Lyotard, Veyne, Koselleck, etc.); second, they force us to ask ourselves whether, despite the principle of play they draw on, these strategies wholly escape ideology, with play also potentially containing the possibility of its own tyranny.

Au printemps de 1995 les étudiants du département de littérature comparée à l'Université de Montréal ont proposé la tenue d'un séminaire collectif autour de Bill Readings pour la rentrée de l'automne. J'ai accepté d'animer ce séminaire en sachant qu'il faudrait éviter de s'en tenir à l'exégèse de l'oeuvre d'un collègue et ami dont les derniers textes étaient par ailleurs encore sous presse. À première vue précipitée, la tenue du séminaire devenait impérative parce qu'il s'agissait d'assurer une certaine continuité entre le travail amorcé par Bill Readings au département de littérature comparée et l'avenir même de ce département au sein des institutions vouées à la crise existentielle: la littérature, la culture et l'université. D'où le titre rapidement convenu pour ce séminaire: Autour de Bill Readings: Littérature, Culture, Université.

Avec un peu de recul, le titre est apparu mal choisi, car la démarche intellectuelle qui motive un tel séminaire ne se laisse pas cerner, comme ce titre le laisse croire, en une série concentrique: Bill Readings pensant la littérature dans la culture dans l'université. Cette concentricité, voire cet effet de centralité qui semble reléguer les participants d'un séminaire aux pourtours d'un cercle et ne les engager qu'à veiller au feu, tels des disciples réunis autour du foyer consolateur, ne concorde en rien avec le type de parcours intellectuel auquel invitent les textes de Bill Readings. Bien qu'inusité, le titre le plus approprié eut rappelé avec humour ce que les amis de Bill connaissaient bien de lui; jouer au billard avec Bill Readings, ou comment observer les déplacements et les disparitions des entités littérature, culture et université . Dans le jeu de billard, comme dans tous les jeux, le joueur est à la fois observateur et partie prenante, sujet et objet du jeu. Jouer avec signifie être autant observateur que partie prenante, reprendre le jeu là où l'autre le laisse - l'adversaire ludique autant que le partenaire. L'oeil du joueur s'exerce d'abord et avant tout à la mobilité du jeu; celui-ci exige en retour 
attention et souplesse. Au jeu de billard, tout bouge et s'entrechoque. Les ricochets sont de rigueur. Le triangle posé sur le tapis au début du jeu n'est destiné qu'à éclater sous le choc circonstantiel opéré par le joueur, mais jamais définitivement contrôlé par lui. Le seul support de contrôle permis à ce jeu ne s'appelle pas sans raison le diable ; cette seule évocation devrait suffire à rappeler aux joueurs que nous sommes que chacune des impasses épistémiques où nous nous trouvons, même dans l'impossible, comporte des ressorts inattendus, et que c'est parfois sur ce caractère inopiné des événements que repose le contrôle.

La pensée de Bill Readings commande l'apport d'autres joueurs parce qu'elle offre une mise. Comme dans tout jeu, c'est ce qui arrive qui intéresse cette pensée, soit l'événement même de ce jeu auquel nous sommes invités dès lors que nous occupons une place, une mobilité dans ce cadre immense appelé l'université . Ce cadre est aussi celui dans lequel sont interpellées, dans un bruit de plus en plus choquant, la littérature et la culture. La question à poser se résume donc ainsi: jusqu'à quel point les intellectuels (dont nous sommes encore jusqu'à nouvel ordre) sont-ils mobiles dans la circulation des savoirs? Devons-nous comprendre cette mobilité (ce désir de traverser les disciplines, de migrer sur les horizons du savoir et d'en explorer la variété des objets) comme notre stratégie ou comme un impératif imposé du dehors? Cette mobilité offre-t-elle aussi la possibilité d'une mobilisation devant les nouveaux marchés de la connaissance? Ou est-ce, au contraire d'une mobilisation, une forme d'abdication devant l'aléatoire des marchés? Autrement dit, cette traversée des apparences, confiée habituellement à la pensée et par laquelle on s'engage de plus en plus à jouer sur les limites identitaires et disciplinaires, doit-elle être reconnue comme une tâche librement consentie ou comme une véritable tyrannie du jeu?

Sommes-nous les maîtres du jeu ou des joueurs en état de flagrante dépendance? Full players or addicted to the game?

Dans son célèbre essai La Crise de la Culture, Hannah Arendt usait d'une image singulière pour disculper les penseurs du XIXe siècle des torts dont on pouvait les accuser une fois l'événement Auschwitz, non pas passé , puisqu'il porte en lui la marque de ce qui justement ne passe pas, mais frappé de sa propre évidence: 
Tenir les penseurs du XIXe siècle, rebelles à la tradition, pour responsables de la structure et de la physionomie du XXe siècle, écrit Hannah Arendt, est même plus dangereux qu'injuste. Les implications manifestes de la domination totalitaire réelle vont bien au-delà des idées les plus radicales ou les plus aventureuses de n'importe lequel de ces penseurs. Leur grandeur réside dans le fait que leur monde leur est apparu comme envahi par de nouveaux problèmes et des difficultés auxquels notre tradition de pensée était incapable de faire face. En ce sens, leur propre sortie hors de la tradition, nonobstant l'emphase avec laquelle ils la proclamèrent (comme des enfants sifflant de plus en plus fort parce qu'ils sont perdus dans l'obscurité), n'était pas non plus un acte délibéré de leur choix.[ $\underline{1}$ ]

Sommes-nous, à notre tour, des enfants qui sifflent trop fort pour ne pas reconnaître cette fois, non pas l'obscurité, mais le bruit ambiant qui, de toute manière, recouvre nos voix? Comme le rappelle Hans Ulrich Gumbrecht dans un récent essai, il nous faut peut-être admettre l'absence d'intérêt public pour nos activités, la marginalisation croissante de notre travail qui ne nous laisse même pas l'initiative de la résistance.[ $\underline{\mathbf{2}}$ ] Gumbrecht réaffirme le caractère inoffensif des études littéraires qui n'ont rien à craindre du système actuel; à preuve: aucune action négative ne s'est interposée devant la menace de notre supposée subversion. Nos concertations dites de résistance laissent froides les instances décisionnelles des institutions du savoir et, plus généralement, ne perturbent d'aucune manière les activités publiques. Dans les faits, elles sont déjà parfaitement intégrées au système. Nous serions donc déjà fantomatiques et les sifflements des fantômes s'harmonisent ici avec l'entreprise de virtualisation que sont devenues les communications humaines.

Il ne s'agit donc plus d'évaluer le prestige d'une discipline comme celle de la littérature comparée, ni même de sonder le prestige de l'université comme institution des savoirs, mais d'interroger ce désir qui nous pousse à réfléchir, discuter et repenser la culture et l'université. Ce désir dépend-il de notre volonté ou nous est-il imposé? Quels moyens avons-nous encore d'être les agents de quelque chose (du changement, du progrès, du savoir, de la culture ou de l'histoire)? 
Au-delà de la fonction des intellectuels dans les sociétés actuelles, de la place de la littérature, du contenu des disciplines et de la restructuration des facultés et des universités, c'est la pertinence de poser les problèmes encore en termes d'institution et d'idéologie qui est mise en doute, semble-t-il, de manière radicale. Cela se vérifie particulièrement depuis la vaste entreprise commerciale que l'on appelle communément la mondialisation, dans le déplacement des territoires et l'effondrement des sites. L'intellectuel ne serait déjà plus une figure publique; il n'y aurait plus de discipline au sein de l'université capable d'incarner à elle seule l'université (comme c'était le cas autrefois de la littérature ou de la philosophie); l'université ne pourrait plus incarner à elle seule la culture, d'où la possibilité de fragmenter les savoirs et de les traverser de part en part. L'université peut désormais faire des Cultural Studies, soutient Bill Readings, parce que la culture ne désigne plus rien et ne représente plus aucune menace à l'hégémonie du système de formation que l'université entend mettre en place. Sous le coup de l'effondrement des États-Nations, le savoir a perdu sa vocation émancipatrice et l'université a sacrifié son Idée unique et transcendante, son identité. Elle a même remplacé sa mission constituante, celle de la transmission de la Vérité, par un caractère de pure efficience. Elle est devenue à elle-même son propre système, bureaucratique, administratif, auto-régulateur et producteur d'un savoir consumériste, commodifié. En s'adjoignant cependant une fausse idée, soit celle de l'excellence, l'université contemporaine persiste à se percevoir comme une institution formatrice essentielle, livrée aux plus hauts espoirs d'une jeunesse à qui il faut vendre du savoir, efficacement, c'est-à-dire vite.

L'excellence, écrit Bill Readings, est purement une unité de valeur interne (...). Elle est le principe intégrateur qui permet de tolérer la diversité sans danger pour l'unité du système. [ $\underline{\mathbf{3}}$ ]

Dans ses grandes lignes, ce constat n'est pas l'exclusivité du travail de Bill Readings. Gerald Graff et Michel Freitag, pour ne nommer que ceux-là, font une synthèse analogue de la situation des universités contemporaines[ $\underline{4}$ ]. Cependant l'analyse faite par Bill Readings nous pousse de manière distinctive au-delà de la stratégie à adopter, vers une mobilité qui nous permet de saisir des éléments dépassant les enjeux immédiats du problème. Devant un problème dont la résolution commande, de manière contradictoire, à la fois une certaine distance et la capacité de coller à la réalité , Bill Readings refuse d'entendre une solution. Il en 
exploite plutôt le nerf: au lieu d'une solution pratique, il en appelle à la diversion heuristique du problème. Je m'explique: à la stratégie de forte résistance (Freitag), aux positions plus pragmatiques (Gumbrecht) et aux désirs de consolation (A. Bloom) ou de réformes (Alain Renaut), Bill Readings oppose une perspective stratégique qui débouche, non pas sur une solution, mais sur un détournement de la problématique. C'est ce détournement (terme dont il use lui-même) qui m'apparaît le plus riche, certes non pas sur le plan politique où il comporte quelques dangers, mais sur le plan épistémologique et heuristique: travaillons donc à pousser à bout la logique du système, semble-t-il dire, car cette logique permet pour la première fois de faire entendre des voix tues jusque-là. En se faisant entendre, ces voix finiront bien par gagner notre confiance; elles finiront bien par miner la complexité du système sans jamais tomber dans la perversité de vouloir la maîtriser; mises à la place des voix mille fois ressassées, elles finiront bien par investir ce vide institué par le phénomène de l'excellence - vide dans lequel il vaut encore mieux placer quelque chose avant que le système ne réimpose une voix monocorde. Mettons-y donc les voix discordantes et irrécupérables (c'est Readings qui le croit), avec leurs intonations impromptues et les différends qu'on y entend. Mettons-y ces voix exclues par l'institution et le projet de la modernité, celles des groupes ethniques, voix non-occidentales, voix des femmes, des gais et des lesbiennes. Tant mieux si le monstre a perdu la tête, car ces voix discordantes réinventeront des têtes suppléantes, une multitude de substituts drolatiques, de talking heads indéfiniment remplaçables. Mettons donc des corps à la place de la tête. Mettons-y tous les corps rabroués depuis l'aube du grand projet moderniste.

En caricaturant un peu, je ne présume pas ces voix imbues de légèreté, purement distrayantes et tout aussi vides que le vide qu'elles viennent combler. Mais en insistant sur une certaine facilité à les concevoir dans le foisonnement et le bruit, sur la légèreté même qu'accompagne leur transgression, on fait voir combien la stratégie adoptée reconnaît à ces voix une vertu ludique. Or ce faisant, cette même stratégie ne risque-telle pas d'user de ces voix comme d'un paravent afin d'assurer le secret, l'indétermination de sa propre posture? Elle écarte en effet pour ces voix la possibilité d'occuper à nouveau le centre, de réclamer pour ellesmêmes une identité, un lieu sans l'aliénation, une vérité et un nom. Ces voix deviendraient donc les simples fairevaloir d'une posture intellectuelle d'autant plus périlleuse qu'elle travaillerait à son propre déplacement. 
On peut certes dénoncer les torts créés par toute quête émancipatrice, en énoncer les travers et en révéler la force d'aveuglement. Mais il est toujours facile de dénoncer pour l'autre, une fois sa propre expérience d'émancipation faite et reconnue, le caractère désuet de toute émancipation. La stratégie intellectuelle visant à mettre à profit la désintégration institutionnelle afin d'en tirer les seules possibilités encore régénératrices ne risque-t-elle pas d'enfermer ces voix dans leurs propres effets de résonnance, dans la redondance des échos qu'elles produisent et de sceller toute tentative d'affirmation volontaire? Ces voix nouvelles, en poussant les disciplines à se discipliner à nouveau - quoiqu'on en pense -, mais vers d'autres fins, ne servent-elles pas d'entremets aux universitaires actuels, entre le moment où la pertinence de la culture s'écroule et celui où l'institution du savoir se confond à la qualité totale de l'économie de marché? Vus sous cet angle, les études gaies et lesbiennes, les Cultural Studies, le féminisme et les études regroupées sous le terme unique de la nonoccidentalité , confinés au paradoxe de leur propre institutionnalisation, sont poussés (n'est-ce pas bien pire?) à l'institutionnalisation de leur faiblesse (the culture of the subaltern). Car on ne veut surtout pas voir ces voix se conformer à la force contre laquelle elles exercent leur dissonnace et s'identifier ainsi à un pouvoir dont on assume mal, par une profonde dénégation, qu'il nous échappe et qu'il nous revienne tout à la fois.

Pour Bill Readings, cette dérive de la dérive, ce retournement de la stratégie contre elle-même semble relever de l'impensable. Le jeu, ou plutôt le coup opéré ici dans le jeu, se trouverait indéfiniment écarté de toute malversation. Le jeu échapperait au piège du pouvoir, de par sa valeur d'utopie, à savoir (littéralement) dans l'impossibilité où l'on se trouve d'assigner à tout jeu un lieu fixe, ou, pour le dire dans les termes les plus proches de ceux évoqués dans Postmodernism Across the Ages[ $\underline{\mathbf{5}}$ ], dans la réitération de l'événement par lequel ces voix s'entrechoquent et qui reste un événement impossible à déchiffrer.

Il y a paradoxe, puisque les voix oubliées risquent, par la perlaboration, de s'oublier elles-mêmes, de perdre leur contenance et de devenir des voix fortes, des voix centrales au lieu de voix dissensuelles et centrifuges. Le seul moyen de surmonter le paradoxe sans le résoudre, propose-t-on, est de se river à l'effet produit: un effet explosif, sublime, radical, un événement impensable. Cet événement n'est concevable pourtant qu'avec le double consentement suivant: d'une part, une forme d'engagement de la pensée critique qui terrorise 
l'institution et de ce fait la déstabilise; d'autre part, la reconnaissance que l'effondrement des institutions s'est déjà produit. D'une manière ou d'une autre, la pensée reste extérieure à l'événement, refusant les pièges de la conscience et de l'omniscience, mais se laisse volontairement impressionner, toucher et marquer par ce qui arrive. Cette pensée n'est plus le sujet terrorisant de l'institution des savoirs, mais le terroriste spontané un travail de l'inconscient -, le hooligan dont l'action erratique ne se réclame plus d'aucune légitimité.

Mais comment ce hooliganisme intellectuel peut-il résoudre le paradoxe par lequel on entend déstabiliser une institution déjà effondrée? Comment terroriser un mort? L'activité du hooligan peut-elle se laisser concevoir avec une telle tranquillité d'esprit, comme un simple violon d'Ingres ?

Il y a ici un problème de chronologie: comment peut-on produire ce qui s'est déjà produit, détruire ce qui est déjà détruit? La stratégie adoptée par Bill Readings emprunte ici à Lyotard la voie la plus insolite mais la plus commode, soit celle qui rabat toute histoire, toute chronologie, sur un caractère d'événementialité pure: pur événement, c'est-à-dire pure production que le penseur critique isole du reste de la trame, pur effet impensable. A ce caractère d'événement, si promptement issu du sublime kantien, on donnera le nom de postmoderne.

Pour paraphraser Lyotard, nous dirions que l'université postmoderne est une institution dans laquelle on reconnaît déjà et trop tard des effets d'effondrement. Le paradoxe n'est paradoxal que du point de vue institutionnel; mais du point de vue des voix oubliées qui ont hanté depuis toujours l'institution sans droit de paraître, il n'y a rien de paradoxal à concevoir la spectralité à la fois comme une constituante de l'institution et une menace pour son intégrité. Le fantôme est par définition un déjà là non manifeste, tout à la fois promesse et héritage. Les voix oubliées par l'institution parviendront toujours de l'extérieur, mais cet extérieur est aussi le topos refoulé de l'institution, l'invisible défaut qu'elle cache à l'intérieur de ses murs. Ces voix sont extérieures à l'université, extérieures à la stratégie elle-même et demandent aux intellectuels de devenir, par ricochet et détournement, extérieurs à euxmêmes, de saborder une fois pour toutes le navire moderne de l'intériorité. Ce faisant, en tant 
qu'intellectuels, nous devons accepter notre faiblesse, $\mathrm{y}$ accéder même, en tout état de cause.

Ce qui s'invente, au coeur de ce qui s'énonce pourtant encore ici comme une théorie, est une résistance par la faiblesse et la faiblesse de la théorie comme force : jouer les petits récits contre les grands; jouer les différends plutôt que les consensus, les différences plutôt que l'identité; endosser la déréférentialisation attribuable au discours de l'excellence afin de jouer la déréférentialisation politique; jouer la lecture comme un acte de résistance, comme le devenant-mineur de la grande littérature, comme le passage de la comparaison à la différence [ $\underline{\mathbf{6}}$ ]. Cette exhortation, cet extraordinaire appel à la mobilité est peut-être la seule issue, non pas tant salvatrice (puisqu'on n'en sort jamais), mais repérable dans un système où la pensée se gère. On opère par allégories, par glissements de figures. Le postmodernisme devient ainsi un autre nom, une autre figure de la faiblesse, une autre figure du même événement toujours prêt à surgir dans le creux de n'importe quel moment et de n'importe quelle littérature de la modernité, comme un fantôme terré depuis longtemps. La postmodernité devient l'exercice stratégique de la modernité contre elle-même. Le sublime devient une figure de l'abjection (une autre expression du rejet), de l'esthétique pour elle-même. L'événement devient l'inconscient fulgurant (un retournement) de la dialectique (donc de l'histoire) par lequel le faible se trouve enfin à figurer.

Je me permets ici un détour par les théories de l'événement. Pour comprendre la position introduite par Bill Readings à l'égard de la crise des universités, il est important de saisir d'où vient la teneur événementielle de la théorie qui la sous-tend, soit la lecture lyotardienne du postmoderne.

Fondamentalement, on peut résumer en trois grandes dichotomies les traits qui émaillent la théorie de l'événement. Outre le couple modernité-postmoderne tant travaillé par Lyotard et repris par Bill Readings, il faut tenir compte de ce que l'historiographie française (Nora et LeGoff surtout) et la théorie de l'événement développée en Allemagne par Reinhart Koselleck ont souligné. Je tiens à revenir sur l'historiographie française et la théorie de Koselleck parce qu'elles sont 
arrivées à cerner les apories sur la base desquelles il est révélateur de lire le postmoderne lyotardien.

Dans le premier cas, il s'agit de la dichotomie entre le caractère d'irréductibilité et de concrétion de l'événement et son caractère de construction ou de fictionalité; dans le second cas, il s'agit de la dichotomie entre structure et récit qui influe sur la définition de l'événement. Chacune de ces dichotomies repose sur la possibilité de relations et de hiatus entre les deux pôles concernés pour chacune d'elle: le hiatus, par exemple, entre l'impossibilité de nier à la fois l'événement d'une guerre et la construction médiatique; ou encore, la relation et la différence par lesquelles l'événement se répète structurellement et se raconte dans sa singularité. C'est ce jeu des hiatus et des relations qui permet d'énoncer l'Histoire, le changement, et c'est à partir de là qu'il convient de saisir le caractère événementiel de la postmodernité entendue par Bill Readings.

Dans un chapitre de Le Futur du Passé[ 7 ], Reinhart Koselleck commence par affirmer la disposition nécessairement narrative de l'événement, avant de nuancer: s'il est vrai de dire, sur le plan épistémologique, que les événements ne peuvent qu'être racontés et les structures décrites, le caractère processuel de l'histoire moderne n'est en réalité pas autrement saisissable qu'à travers l'explication des événements par les structures et inversement. [ $\underline{\mathbf{8}}$ ] Bien sûr, les événements peuvent se définir comme des entités de sens qui se laissent raconter - ainsi en est-il des témoignages vécus et transmis jusqu'au XVIIIe siècle. Reprenant à son compte l'énoncé de principe de Simmel, Koselleck convient de ce que ces unités de sens exigent, dans leur constitution même, un minimum d'avant et d'après. Mais cette contrainte de la chronologie n'empêche pas de concevoir l'histoire dans sa dimension structurelle, ce dont la nouvelle histoire se chargera en opposant à l'histoire dite événementielle (celle des guerres et des révolutions) une histoire de longue durée (Braudel). Cette dernière modifie la teneur narrative de l'histoire en lui confiant une exigence essentiellement descriptive; les lentes transformations, ces plages mouvantes et ces entrecroisements d'états de choses lentement mis en branle font se diluer la chronologie au profit de grandes transformations structurelles. Ces transformations laissent prise uniquement à la description des rapports de production, des modes de domination, des structures constitutionnelles. Le récit historique cède à la représentation des structures de l'histoire. Mais les deux 
niveaux, celui de la structure et celui du récit, restent incompréhensibles l'un sans l'autre; ils restent complémentaires et échangent même leur place selon la question soulevée [ $\underline{\mathbf{9}}$ ], selon l'intrigue choisie, dirait Paul Veyne[ 10 ]. Koselleck arrête son analyse précisément sur ce qui s'avère tenir à la fois du hiatus et de la relation étroite entre la structure et l'événement dont une aporie méthodologique empêche l'amalgame parfait. Sans cette double et difficile relation entre la structure et l'événement, la catégorie du possible échapperait entièrement à l'histoire, de même que l'écriture de l'histoire serait impensable, car autant il n'est de langage approprié pour rendre compte de chaque événement dans sa singularité, autant tout événement établi et présenté historiquement vit de la fiction de sa facticité, la réalité elle-même [étant] passée. [ 11 ] L'interaction entre le niveau structurel et le niveau événementiel de l'histoire offre les conditions de possibilité des histoires. En assumant le caractère hypothétique de la réalité des événements et le caractère fictionnel des événements racontés, ces concepts thématisent des traits contemporains dans le non-contemporain qui ne se laissent pas réduire à la simple succession du temps de l'histoire. [ 12 ]

Sur ce dernier point, il faut revenir aux travaux amorcés dans la mouvance de la nouvelle école française par Pierre Nora et Jacques LeGoff pour saisir dans toute sa portée la force de l'aporie méthodologique dont parle Koselleck. En effet, les travaux de ces historiens montrent combien la limite entre la concrétude de l'événement et sa construction reste fragile; en d'autres mots: nous n'avons pas de terme pour différencier l'événement construit de l'événement réel. Dans les débats entourant cette question, les arguments sont tout aussi percutants en faveur de la concrétude indélébile de l'événement qu'ils le sont en faveur de sa valeur de construction . Le domaine de l'événement est-il celui de la factualité ou celui de l'effet ? Et encore, quelque chose tiraille ici, entre l'effet entendu comme une effectivité (héritage de l'empirisme) et comme affectivité (credo postmoderne).

Même en cherchant à concilier les deux postulats (empiriste ou postmoderne), on se trouve à osciller d'un pôle à l'autre. D'un côté, on souligne l'irréductibilité de l'événement entendu comme trace, comme marque d'un réel indéniable. L'événement est ce qui fait saillie, et nonobstant la difficulté à en percevoir les limites, il reste ce qui de soi saute à l'évidence. Une blessure, une douleur, une rupture, l'usure dans les édifices, la radicalité indéniable des inscriptions, des différences et 
des morts offrent au temps une possible unité de mesure, un marquage. À la limite même, l'événement devient le synonyme du réel, la preuve qu'il s'est bel et bien produit quelque chose. Mais d'un autre côté, l'événement désigne quelque chose qui advient, donc qui passe, et non simplement ce qui est. Il témoigne ainsi de la fluidité et de l'éphémère plutôt que de l'assurance des choses. L'événement, de surcroît, est le produit d'un consensus, car il reste désigné dans et par le langage dont use un groupe communicationnel; il est le point de jonction des histoires racontées, le point de départ de la fable. Au rythme de l'information qui passe et de la mémoire qui oublie, les événements sont, pour le dire comme LeGoff, des données faussement réelles .[ 13 ] Dans le monde gonflé par la fabrication de l'événement et son enchaînement perpétuel, l'artifice est la vérité du système [ $1 \mathbf{4}$ ]. Et pour le comble, étant le produit d'une réalité toujours elle-même travaillée par le mythe, l'événement est aussi le fruit de l'anticipation et de l'attente, la venue du Messie comme l'annonce d'une catastrophe.

Partant de ces mêmes apories, Jean-François Lyotard va s'employer à faire valoir le choc incommensurable entre la concrétude de l'événement et sa construction, entre ce qu'il désigne comme étant deux temps bloqués : le temps de l'histoire et le temps de l'écriture de l'histoire qui sont intriqués l'un dans l'autre, bien que foncièrement rupturés. C'est que l'événement est la production d'une singularité radicale qui ne peut plus être représentée par une histoire générale sans perdre sa singularité, sans être déviée et se réduire à un simple moment . Tout événement, selon Lyotard, ne peut être compris au moment où il arrive, parce que sa singularité est étrangère au langage et à la structure de compréhension qu'il produit. Par conséquent, l'événement ne saurait se laisser réduire à une unité de chronologie, à un moment. Il excède son cadre référentiel, déplace son cadre. Après lui, rien n'est plus pareil. Le méta-événement à la recherche duquel Lyotard semble déployer toute son énergie est censé désamorcer le métarécit qui le gêne; en rupture épistémique avec le métarécit, Lyotard inscrit le choc incommensurable de l'événement conçu sur les ruines du sublime kantien. Il s'agit en fait d'une double incidence: celle du sublime kantien et celle d'une chaologie qui s'ignore, très proche du fulgurant effet papillon, puisque l'infiniment petit bouleverse cette fois l'infiniment grand. L'événement devient, pour Lyotard et Readings, une déflagration, un effet, une force de figuration, un enchaînement de suppléments . D'une 
certaine manière, on joue ici, sur la trame d'une histoire en crise, le choc de l'instant contre l'ordre du moment .

Sur le plan méthodologique, la manière de régler les grandes dichotomies de l'événement correspond donc ici à une sorte de recours à la posture théologique. Celle-ci consiste à donner à une figure (et non plus à un concept ni même à une catégorie) dont la force dépasserait l'entendement, non pas la capacité de représenter l'ensemble des relations et des hiatus impliqués par ces dichotomies, encore moins celle d'en expliciter les termes, mais le pouvoir de les sublimer.

Par ce moyen, on procède au court-circuitage des relations entre structure et récit, entre concrétude et construction. Ce court-circuitage opère en quelque sorte le renversement de tous les termes engagés dans ces relations. Le résultat est spectaculaire. L'événement, même au sens narratif du terme, devient ce qui ne se laisse pas raconter, car les petits récits sont tus. Et puisque le postmoderne participe tout à la fois de l'avant et de l'après, il est par définition ce qui hante (présent et absent à la fois), une sorte de longuesingularité paraphrasant la longuedurée proposée par la nouvelle histoire. S'il y a quelque chose qui se rapproche de la longuedurée, soit les modes de domination et les rapports de production, ce ne peut être, en ce qui concerne Lyotard, que ces micro-mobiles producteurs de différends, soit d'éléments non-comptabilisables, distincts en tous points de la grande hégémonie du changement qu'est le progrès moderne. Le récit devient ce qui ne se raconte pas mais se laisse décrire par la seule figuralité de ses effets. Le potentiel de narrativité de l'événement est englouti par l'irréductibilité de l'écriture de l'histoire; au lieu de la factualité même de l'événement, c'est à l'écriture que revient le caractère d'irréductibilité, de sorte que ne se raconte que l'écriture elle-même, sa répétition. Le même type de renversement s'opère sur tout groupe communicationnel instruit par la fulgurance de l'événement: au lieu de se voir unifier, bonifier par l'événement, la communauté est rupturée par le choc produit. Ainsi, selon Lyotard, l'événement est impossible à désigner dans le langage, à traduire dans la communication, puisqu'il vient précisément rompre les conditions de possibilité d'un consensus nécessaire à sa propre désignation. On comprendra que la figure y est entendue comme un ordre de rupture (une métonymie, une anamorphose) et non comme une opération de synthèse, comme c'est le cas par exemple chez Paul Ricoeur. 
L'effet de l'événement est à la fois singulier et interminable (donc nonlocalisable). L'événement postmoderne n'offre donc pas la possibilité de marquer le temps, de suivre la chronologie, mais l'ultime possibilité de marquer la marque: le marquage de l'écriture de l'histoire qui n'est rien d'autre à son tour qu'un marquage, soit un événement. Pour inverser et paraphraser Pierre Nora, nous dirons que Lyotard affirme, non pas que l'artifice est la vérité du système, mais que la vérité est l'artifice du système.

Le jeu des relations et des hiatus s'est transformé en répercussions sans fin, en violentes détonations: le hiatus produit un autre hiatus, et ainsi de suite. On ne sera pas surpris de voir se constituer chez Lyotard une théorie du différend à la base de laquelle Auschwitz désigne l'événement limite. En définitive, la mort devient un modèle pour l'événementialité, car les morts ne peuvent témoigner de la mort qu'ils sont pourtant les seuls à connaître, de ce que cette connaissance équivaut à un non-savoir. La mort est le hiatus des hiatus et Auschwitz désigne l'incommensurable.

Cependant on peut se demander en quoi cela rend nécessaire une théorie de l'événement dans les termes du postmoderne. Pourquoi cette urgence de penser la spectralité et la hantise, la mort et l'immoral dans les termes d'une longuesingularité (sic) qui aurait paradoxalement l'effervescence, l'effet et l'impact d'un instant fulgurant? La mort n'a jamais été l'exclusivité d'une tension entre la modernité et le reste. Et s'il faut bien sûr rendre compte d'Auschwitz en y braquant le sort et la responsabilité de la modernité, le renvoi au caractère d'instantanéité muette de l'événement ne risque-t-il pas de faire s'effondrer toute mémoire? Jusqu'à quelles limites peut-on soutenir l'inexplicabilité d'Auschwitz? Est-il pour autant nécessaire d'emblématiser le temps par le truchement d'une figure (le postmoderne) dont l'effet spectaculaire ne peut, à long terme, que s'atténuer? D'une modernité basée sur le principe téléologique de l'Histoire, nous passons à une condition postmoderne où le principe d'immanence régit l'événementialité: l'événement comme immanence, voilà la condition postmoderne qui n'aurait par conséquent aucune assise chronologique. Le postmoderne, affirme Bill Readings à la suite de Lyotard, est l'autre de la modernité - son altérité latente, lovée, toujours prête à rompre le secret de sa spectralité dans les textes, dans l'écriture de l'histoire et dans la littérature. La littérature n'offrirait pas autre chose que les traces de cette figuralité, ses effets de lecture. 
Si le postmoderne est l'autre de la modernité, tout engagement critique et théorique devant cette irréductible force événementielle amène donc à considérer la littérature et la culture dans un constant porte-à-faux avec la modernité, comme si finalement, tout se mesurait, une fois de plus, à l'échelle de la modernité - ce qui est loin de nous expulser du cadre moderne comme on le prétend par ailleurs. Si le postmoderne se contient dans l'événement seul et le déborde tout à la fois, comment saurait-il donner prise à une histoire dont la temporalité est plus complexe qu'un agrégat d'effets répétitifs? On aura beau s'empêcher d'user encore du terme d' histoire, le refuser transgressivement, il reste que le propre de cette transgression nécessite la reconnaissance d'une limite; il reste aussi que nul ne peut se dire en-deça d'une historicité sur les fondements de laquelle, de toute manière, il est appelé à critiquer. Si l'histoire nous aliène, la reconnaissance même de cette aliénation bonifie en quelque sorte le recours à la catégorie de l'histoire, lui donne raison. Au-delà comme en-deça de cette posture, on cesse de parler.

Le renversement spectaculaire opéré par Lyotard sur la question de l'événement et le détournement proposé par Bill Readings comme la stratégie appartenant désormais aux nouveaux hooligans que seraient devenus les universitaires font-ils vraiment l'économie de l'histoire en recourant à la figuralité de l'événement? Une ambiguïté demeure quant à la portée même de ces discours: leur portée éthique détonne sur leur valeur stratégique - on parle tout à la fois de l'impossible témoignage des victimes d'Auschwitz et de l'impossible assignation d'un sujet dans le jeu du libre marché. Une telle coïncidence entre l'holocauste totalitaire et la logique du néo-libéralisme, entre le système qui nie toute subjectivité et la stratégie appelée paradoxalement à le miner devrait être analysée plus avant. Quelles sont les implications d'un jeu qui s'accommode si facilement du totalitarisme? Comment la stratégie peut-elle atteindre une certaine efficace si cette efficace est la condition même du système en rupture avec lequel elle prétend agir?

En investissant le vide idéologique de l'excellence, le hooliganisme universitaire ne vise à rien d'autre qu'à reprendre sans cesse ses propres jeux de figuration, les effets de sa propre événementialité - sans hiérarchie, 
sans ordre et sans objectif extérieur à lui-même. Mais ce faisant, comment saurait-il justement s'intéresser à autre chose qu'à lui-même, c'est-à-dire à quelque chose qui tienne de l'Autre au nom duquel il se réclame néanmoins? Soit il se condamne alors à l'exploration narcissique de sa propre événementialité, soit au contraire il s'annihile en instruisant son propre procès où, comme dans tout procès instruit par l'accusé luimême, la culpabilité est tout aussi fondée que l'est le manque de légitimité du juge à assurer la justice. Dans le contexte politico-économique actuel, rendre l'université aux têtes parlantes, donner à toutes les voix discordantes, non pas une place qui leur revient, mais la simple possibilité de danser sur la scène ruinée des savoirs, c'est constuire une anti-socialité, un hooliganisme au nom de la reprise constante de chacune des figurations: figuration avant toute chose de ce qui se tait et qui, autrement dit, n'a pas de voix . À défaut d'être présent, ce que l'université avait écarté en qualité d'institution moderne par excellence, fait désormais fureur comme autant d'effets produits grâce à l'institution et contre elle. Pendant ce temps, ce qui continue à parler, soit le discours qui persiste à attirer l'attention sur ces effets, ne peut faire autrement que s'enfermer sur lui-même. Ainsi entend-on beaucoup parler de la littérature comme de l'autre de la philosophie phallogocentrique, mais n'entend-on que très peu cette même littérature parler de sa propre voix. Sans doute est-ce parce que la littérature se révèle ellemême comme un discours apte à penser, à la fois dans les marges de la philosophie et en-dehors de l'institution philosophique. Mais sans doute est-ce aussi parce que la littérature est le terrain le plus glissant où la terreur et le jeu s'entraînent le mieux ensemble. Le hooliganisme y a déjà son histoire. Et le hooliganisme se refuse d'admettre qu'il a une histoire.

L'excellence est le symptôme d'une université aux prises avec la désinstitutionnalisation. Elle indique que l'université est maintenant passée du statut d'institution à celui de marché. Le cadre de la gestion dans lequel les universités contemporaines opèrent désormais n'est pas un choix d'institution, mais la conséquence de ce que le régime politique dans lequel elles sont appelées à persévérer est fondamentalement celui de la gestion: gestion des ressources, des biens, des personnes et des déchets. Cela est, parce que l'accroissement des richesses et l'exploration matérielle ont trouvé leurs 
limites. Nous gérons dorénavant les choses. Ce régime est lui-même hautement ludique et fondamentalement économique. C'est un jeu sérieux contre lequel le jeu d'un événement, l'intrusion d'une occurrence ludique différentielle ne peut hélas pas grand chose. Aussi, la stratégie qui se rapporte à cette différentialité peut-elle prétendre à une posture éthique, à faire autre chose que du politique, elle n'en sera toujours que ravalée par cette capacité de l'économie de marché à intégrer toute valeur différentielle.

Bill Readings a judicieusement saisi l'urgence de repenser l'université et la culture dans les termes d'une économie. La pertinence de poser le problème dans les seuls termes d'une crise institutionnelle identitaire ou d'une crise des idéologies fait défaut. Mais le problème reste à savoir comment ruser avec les économies actuelles, avec l'ordre désordonné du marché, sans se soumettre entièrement à la logique du marché - la loi $d u$ marché n'étant pas autre chose que l'infini désir du jeu. Dans ce contexte, le marché est appelé à devenir ce qui reste après la dissolution économique des institutions, leur transmutation en pures économies. Le marché se trouve alors à reposer sur le double principe de la variété (diversité, compétition, nouveautés) et de l'hégémonie (plus rien ne lui échappe, rien n'étant plus en compétition avec le marché lui-même).

Dans cette perspective, la culture ne se trouve plus nulle part, non pas parce qu'elle est absente, mais parce qu'elle est partout - tout étant devenu culturel - même la nature désignée de nos jours comme le patrimoine terrestre. Elle ne constitue plus un discours de légitimation - et là-dessus Lyotard avait raison dans son rapport sur la condition du savoir dans les sociétés les plus développées (1979) de décrire les sociétés développées comme celles où l'enjeu n'est pas la vérité, mais la performativité [ $1 \mathbf{5}$ ]. Mais puisque ces sociétés sont déjà ludiques, comment penser pouvoir en miner le jeu par d'autres jeux (coups) sans oblitérer toute possibilité de distinguer (de faire les différences et de saisir les différends) entre les bons coups et les mauvais coups, entre les jeux qu'il faut soutenir et ceux qu'il faut dénoncer? Est-ce à dire qu'il n'y a plus en ces jeux ni souffrance, ni contraintes, ni injustices? Si toute légitimité tombe en désuétude, comment encore parler d'injustice?[ $\underline{16}$ ]

En tant qu'intellectuels, comment résister au vide et se satisfaire d'une simple mobilité dans le savoir si la mobilité est d'emblée la loi du système? Comment désirer encore quelque chose en toute spontanéité si les 
désirs sont engagés d'avance sur les cours du marché? Cette mobilité à laquelle on voudrait confier les dernières tâches de la pensée - d'une pensée pratiquant l'auto-dénégation - est-elle du ressort de notre propre liberté de joueur ou du ressort de l'économie dans laquelle nous nous trouvons et hors de laquelle il n'y a point de salut? Ne sommes-nous pas tristement assignés au jeu, obligés à jouer, livrés au jeu avec pour seules alternatives la voie de la dépendance aveugle ou celle du fair play?

Ce problème est un enjeu majeur dans les écrits de Bill Readings, une figure jamais nommée, celle-là. Et j'ajouterai que c'en est le seul écueil - non pas que la chose, étant irrésolue chez lui, rende inutile son argument, mais le fait de ne pas savoir, et même de ne pas vouloir savoir est compris dans le travail de Bill Readings comme une stratégie politique, comme l'ultime mouvement encore permis à la surface des savoirs. L' événement, par lequel quelque chose advient à travers la dénégation pratiquée par les savoirs modernes, s'autoprésente comme l'instant d'un non-savoir, mais un nonsavoir qui, contrairement à l'ignorance, est assumé. Savoir que je ne sais pas devient le mobile d'une visée essentiellement pédagogique, généreuse et plus compatissante que Bill Readings n'ait bien voulu l'admettre. En effet, cette stratégie est aussi fondée sur une déconstruction du motif chrétien: on en a contre la rédemption promise par l'Université et contre une communauté rassemblée autour du cadavre culturel à dépecer. Et pourtant, la stratégie pédagogique proposée par Readings implique une synergie, certes locale et ponctuelle puisqu'il s'agit de coordonner les efforts dans la classe, mais néanmoins absorbante et exigeante. On sera étonné sans doute de cette disproportion entre une solution aussi ténue, reposant essentiellement sur l'éthique pédagogique, et un problème aussi énorme (rien de moins que la modernité dans ses fondements épistémiques et éthiques). En s'attaquant de front au gigantisme même du problème, Bill Readings pouvait voir sa stratégie se rompre au mur de l'utopisme. C'est cependant par évitement, par détournement, qu'il ouvre une perspective intéressante, allant non plus tant audevant de l'aporie elle-même, mais dans les pores qu'elle offre au regard du non-initié. Retourner la communauté des initiés en théorie des errants, et poser le problème de la communauté des non-initiés, voilà en d'autres termes à quoi se résume l'esprit de cette pédagogie. La pédagogie: un travail dans la classe qui embrasse et chasse tout à la fois l'esprit de communauté, un travail qui lie l'effet du groupe, en sa cohésion même, à sa dimension heuristique, événementielle, éphémère. La 
force de cette pédagogie est d'ignorer précisément sa force. Elle ne peut ni ne veut se prendre pour un nouveau pouvoir, car le pouvoir est investi ailleurs et l'enseignement récolte des restes. Partant de Mai 68, on entrevoit ici la configuration parfaitement mobile des rapports entre l'enseignant et l'étudiant fondés sur la communauté des différences, des différends, celle du non-consensus où l'étudiant, comme l'enseignant d'ailleurs, doit faire l'expérience d'une certaine opacité et d'une certaine difficulté - lesquelles n'ont rien à voir avec les épreuves qu'exigent l'émancipation et le progrès progrès.

Cependant, la volonté de ne pas reconnaître dans le jeu ludique la possibilité du jeu tyrannique (lorsqu'on est abusé par le jeu) est entendue ici comme une stratégie de détournement, un espace dans lequel puisse encore être pensée une certaine subversion, une certaine résistance. C'est sur ce caractère de résistance que l'analyse achoppe. Comment l'intellectuel, alors même qu'on le suppose en train de disparaître, peut-il se convaincre de la maîtrise qu'il peut encore avoir sur sa propre figuration sur la scène du savoir? Comment penser la résistance dans de telles conditions?

Dans cette situation où nous avons l'impression que les jeux sont faits et qu'on ne peut plus jouer avec les jeux sans être aussitôt récupérés par eux ou jouer avec le flou institutionnel sans être récupéré par lui, la tentation est forte d'avouer que l'heure est grave, mais déjà passée. C'est pourquoi sans doute le sifflement de l'intellectuel contemporain, même du fond de l'obscurité, a parfois ceci de burlesque qu'il se rapproche du sifflement que commet le coupable lorsqu'il feint la légèreté.

Même si la stratégie préconisée par Bill Readings me semble de loin la plus rusée, elle me rappelle un exemple assez troublant: celui de la dissidence sous le régime soviétique. Malgré l'énorme différence existant entre les deux systèmes, soit celui de l'économie postmoderne et celui du totalitarisme soviétique, les deux stratégies adoptées pour résister à l'hégémonie du système sont très proches. Dans sa visée éthique, la stratégie de résistance qu'implique l'analyse faite par Bill Readings est très proche de celle dont a usé la dissidence. Cette stratégie de la communauté non-consensuelle s'apparente à la dissidence dans la mesure où toutes deux se refusent à revendiquer le pouvoir, et ce, au nom d'un impératif plus éthique que politique: questionner le 
fondement même du pouvoir en le déstabilisant, non pas par la violence directe, mais par la rupture locale et le mimétisme. Dissidence veut dire littéralement: à-côté, d'où la stratégie qui en découle et qui s'appuie sur le détournement et le déplacement du pouvoir par luimême. Cette posture de l'à-côté, cette mobilité par laquelle on décide de jouer le jeu de l'adversaire afin de le miner de l'intérieur fonctionne. Readings a raison de penser que les tenus à l'écart, les rabroués du système vont finir par refaire surface et disloquer le Tout. Les dissidents avaient aussi raison de croire que le système allait s'effondrer de lui-même, par la force même de ses exigences économiques et l'extraordinaire automatisation de son fonctionnement. Mais voilà, l'exemple soviétique a montré qu'il y avait un prix à payer à l'effondrement, que cette stratégie ne devenait opératoire qu'à la condition d'une terreur que semblaient écarter jusque-là les dispositions ludiques des stratèges: le sacrifice de ses principaux agents. On sort ici d'une logique de la rédemption pour entrer aussitôt dans la mécanique sacrificielle. Après la perestroïka, ces dissidents qui ont miné le système ont trouvé le même sort que les poètes révolutionnaires des années vingt sacrifiés à l'autel de la révolution. Mais cette fois, c'est dans l'indifférence la plus totale et l'oubli le plus anti-héroïque que le sacrifice a eu lieu.

Politiquement parlant, cette stratégie de la dissidence n'a fait qu'écrouer ses principaux médiateurs et permettre le retour - comme si rien ne s'était passé d'une autre brutalité.

A refuser de concevoir le jeu comme étant aussi, possiblement, l'enjeu d'une idéologie, en concevant la fin des idéologies comme un fait accompli et en refusant de comprendre l'économie dans sa teneur idéologique, ne coupe-t-on pas tout recours, ne risque-t-on pas de rester en plan, divisés et manipulables? Comment concevoir en effet la différence entre cette mobilité stratégique et la mobilité dans laquelle on se trouve idéologiquement poussé? Le jeu peut aussi jouer la carte du totalitarisme - et sans dire que c'est le cas actuellement, j'insisterai sur la facilité avec laquelle Jean-François Lyotard enferme, chacun de son côté, le jeu et la terreur, comme si l'un évacuait nécessairement l'autre. Selon lui, la terreur se trouve hors des jeux de langage, puisque l'efficacité de la force procède alors tout entière de la menace d'éliminer le partenaire, et non d'un meilleur coup que le sien. [ 17 ] Or, on le sait depuis l'Inquisition et on le reconnaît sur ce parcours menant de la Révolution à la dissidence, la terreur peut très bien se fustiger elle-même; elle procède tout autant de l'auto-flagellation et de l'auto-harcèlement. Si l'on 
reprend le paradigme du jeu, on dira que le joueur peut passer très subtilement, sur le coup et sans distinction aucune, de la décision volontaire à la tyrannie du jeu. Un coup joué est parfois aussi un coup auquel le joueur se soumet dans la dépendance la plus totale. C'est là la tyrannie de l'intoxication ludique.

Dans le contexte de l'émergence des nouvelles disciplines pour lesquelles l'indisciplinarité est la nouvelle norme, on peut se demander si la terreur ne trouve pas sa voie, ironiquement, à même l'insatiabilité des désirs en jeu dans la recherche toujours plus intense des micro-localités et des différends. Ironiquement, c'est à force de refuser de jouer le jeu du pouvoir et de l'autorité que ces nouvelles disciplines encouragent parfois à leur tour, mais à leur corps défendant, l'exercice d'une discipline de fer, un certain autoritarisme vis-à-vis ses propres exigences.

La lecture lyotardienne du postmoderne sous-tend l'idée selon laquelle tout joueur a le jeu à sa disposition, comme un ressort dont il dispose, en même temps qu'il s'en trouve nécessairement rejeté en tant que sujet. Ce ressort est à la fois ce qui jette l'homme - au sens humaniste du terme - à côté de lui-même, et l'instrument qu'une subjectivité persiste toutefois à manipuler sans qu'on n'arrive à saisir d'où vient cette subjectivité. Il s'en trouve la confusion la plus totale entre le jeu comme stratégie et le jeu comme effet sur lequel le joueur n'a aucun impact. De la pensée, nous passons à l'effet sublime sans même que cette différence ne soit rapportée comme un hiatus important. Le jeu semble, chez Lyotard, toujours égal à lui-même, sans échelle: du jeu toujours égal au jeu, seulement parfois différent, mais jamais ni mieux ni pire, ni bon ni cruel. Or les jeux cruels existent et sont les plus efficaces, les plus mobilisateurs parce qu'ils exigent du joueur comme du public une dépendance totale et une complète identification. Le jeu peut rendre fou, et l'esprit ludique n'a jamais empêché l'idéologique. En ce sens, la modernité se fonde déjà sur l'introduction d'un certain jeu dans l'ordre, d'un certain hasard et d'un amour du hasard cultivé, pour ainsi dire, dans le dos des Dieux.

En faisant s'accorder le jeu avec l'impossibilité d'établir des consensus, Lyotard stipule que les jeux de langage échappent aux règles. Or les jeux de langage n'échappent pourtant pas à cette condition sine qua non des jeux qui veut que chacun d'eux s'appuie sur des règles. Sans la règle, point de jeu. Et ces règles s'appuient à leur tour nécessairement sur un consensus. 
La communauté nonconsensuelle proposée par Bill Readings, par définition, ne tient pas - il en est parfaitement conscient. En cela, elle n'échappe pas au jeu de l'idéologie et offre toujours la possibilité de se voir récupérer par un type de pouvoir ou par un autre. Elle ouvre également la voie, ni plus ni moins que les autres, à la terreur. Dans quelle mesure en effet cette stratégie atomisante évite-t-elle de provoquer sa propre tyrannie? En quoi se démarque-t-elle des autres versions de la légitimation des "droits individuels"? En quoi l'événement par lequel toute communauté devrait s'autominer ne deviendrait-il pas tyrannique et fascinant à son tour?

Il faut revenir au caractère précisément ponctuel de la stratégie pédagogique offerte par Bill Readings pour saisir, dans cette valorisation du dissensus et la difficulté d'en concevoir une possible terreur, le propre d'une situation à laquelle la pensée ne peut plus échapper: particulièrement depuis la fin de la guerre froide, les conjonctures actuelles sont le résultat d'un effet de vitesse sans précédent, à savoir le repliement des tensions idéologiques sur l'hégémonie ludique du marché. Les grandes axiologies politiques et idéologiques ont été vidées de leur sens, confiant à l' éthique une tâche qui la dépasse. Pourtant, si la nappe fut retirée de la table sans que les couverts n'en soient apparemment bouleversés, les convives n'en sont pas moins à se demander encore ce qui s'est réellement passé. Nous en sommes là à examiner les restes sur la table des idéologies. Nous n'avons pas encore trouvé le moyen de penser l'événement d'une disparition qui met en doute notre propre capacité à saisir les choses. La brutalité et la rapidité avec lesquelles la mondialisation semble devoir s'instaurer et modifier considérablement l'enjeu des cultures actuelles et passées nous plongent dans l'interrogation; elles nous forcent à reconnaître le non-savoir au-delà duquel nous hésitons à aventurer la pensée, de crainte de soulever à nouveau - comme dans un perpétuel enchaînement au progrès - de nouvelles brutalités.

Dans cet entre-deux où la pensée se trouve désormais à réfléchir sur elle-même, où elle se sait encore ancrée aux fondements opaques du passé mais attentive à l'urgence de l'instant présent, aucune solution ne saurait encore prendre le relais. Nous commençons à peine à saisir l'étendue du changement à apporter à nos méthodologies. Le changement lui-même ne peut plus être entendu comme un processus homogène. C'est 
pourquoi la solution ne peut être que temporaire et ponctuelle; elle ne peut qu'engager partiellement les choses et avouer sa faiblesse.

Dans une certaine mesure, à l'heure où les experts de la littérature s'occupent de moins en moins de littérature, ces derniers sont en passe de devenir les "nouveaux romanciers" en cherchant à déployer l'intrigue de leur propre disparition dans la prolifération des savoirs et la mobilité des identités. À ce sentiment de spectralité s'ajoute l'étrange nécessité de recourir à une forme de clôture. En "bons romanciers", ceux qui tentent encore de penser et d'enseigner la discipline de la littérature ne peuvent résister à la tentation de résoudre un problème, quand même cette solution passerait par l'infini détour de la fiction. Essentiellement, nous pensons pouvoir et devoir être encore là, ne serait-ce que sur l'horizon de la fiction, pour penser ce que la littérature pense sans nous, à savoir que la terreur et le jeu ne sont que les deux versions inséparables du désir. La littérature, cette chose dont on est censé s'occuper et qui se déplace maintenant et s'effondre peut-être (dehors comme dans l'université), a longuement ruminé ce problème auquel nous sommes confrontés: la littérature est un savoir qui est à lui-même son propre savoir. Dans cette mesure, elle ne s'enseigne pas. On n'enseigne pas la littérature comme on enseigne la philosophie, la musique ou les mathématiques. Enseigner la littérature nécessite la reconnaissance d'une gratuité, d'une posture de l'à-côté: La tâche de l'enseignant est ici gratuite au sens où la littérature se passe facilement de lui. La pédagogie rejoint bizarrement l'épistémologie de la littérature. Peut-être est-ce encore le seul moyen de tromper l'attente dans laquelle nous sommes plongés: revenir à cet accident par lequel un savoir, tout en s'instituant, tout en devenant idéologique, secrète encore la possibilité d'un geste gratuit.

\section{NOTES}

1. Hannah Arendt, La Crise de la Culture, 1954, traduit de l'anglais par Patrick Lévy, Gallimard, 1972, p. 40. 
2. Voir H. U. Gumbrecht, "The Future of Literary Studies?", New Literary History, no. 3, vol. 26, 1995, p. 508.

3. Bill Readings, "University without Culture?", New Literary History, op. cit., cette position doit bien sûr beaucoup à celle développée par Jean-François Lyotard dans La Condition Postmoderne, Minuit, 1979.

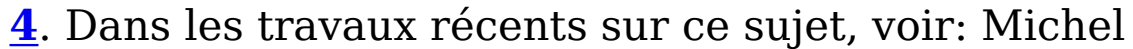
Freitag, Le Naufrage de l'Université, Québec/Paris, Nuit Blanche et La Découverte, 1995; Alain Renaut, Les Révolutions de l'Université: essai sur la modernisation de la culture, Paris, Calmann-Lévy, 1995; le numéro de la New Literary History, op. cit. dans lequel Gerald Graff réplique à Bill Readings.

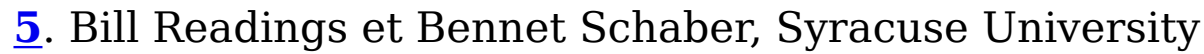
Press, 1993.

6. Bill Readings, Translatio et littérature comparée in Surfaces, Vol.I, 1991.

7. R. Koselleck, Geschichte - Ereignis und Erzählung, Munich, 1972; ici en traduction française:

"Représentation, événement et structure" dans Le Futur du Passé: Contribution à la sémantique des temps historiques. Ed. Ecole des Hautes Études en Sciences Sociales, Paris, 1979.

8. Ibid., p. 138.

9. Ibid., p. 137.

10. Paul Veyne, Comment on écrit l'histoire. Essai d'épistémologie., U.H. Seuil, Paris, 1971.

11. Op. cit. p. 140.

12. Ibid., pp. 140-141.

13. Jacques LeGoff, Histoire et Mémoire, Paris, Gallimard, 1988.

14. Pierre Nora, Le Retour de l'événement in Faire l'Histoire, 1974.

15. Lyotard, op.cit., p. 76.

16. Là-dessus, je réitère la position critique énoncée par Eduardo Galeano dans un article cinglant paru dans Le Monde Diplomatique en janvier 1996, Technologies et mensonges - Vers une société de l'incommunication? : ... 
la notion d'injustice elle-même, naguère une certitude universelle, s'est à peu près estompée jusqu'à disparaître. Le code moral de cette fin de siècle ne condamne pas l'injustice, mais l'échec. (p. 16). Les cas flagrants de la guerre en Bosnie et de la destruction systématique de la Tchéchénie le montrent bien.

17. Op. cit. p. 76.

Accueil Surfaces | Table des matières | Recherche Surfaces Home Page | Table of Contents | Search

PUM | Livres | Revues | Publications électroniques | Vente et distribution 